\title{
PREDICTORES PSICOSOCIALES DEL CONSUMO DE TABACO EN ADOLESCENTES: EXTENSIONES DE LA TEORÍA DE LA CONDUCTA PLANIFICADA
}

\author{
JOSÉ BERMÚdEZ Y ANTONIO CONTRERAS \\ Facultad de Psicología, Universidad Nacional de Educación a Distancia, Madrid
}

\begin{abstract}
Resumen: En una muestra de 227 adolescentes, esta investigación estudió, primero, la utilidad predictiva incremental de la similaridad al prototipo, una vez controladas las variables de la teoría de la conducta planificada (TCP) y, segundo, la contribución predictiva diferencial de los dos componentes del control conductual percibido: expectativa de autoeficacia y anticipación de dificultades. Los resultados evidenciaron que: 1) la intención y consumo de tabaco fueron predichos por las variables TCP (salvo norma subjetiva). 2) La similaridad al prototipo mejoró significativamente la predicción de intención y conducta. 3) Ambos componentes del control percibido predijeron la intención, pero sólo autoeficacia predijo la conducta. Globalmente considerados, estos resultados: son consistentes con la investigación realizada desde la TCP; muestran el incremento en capacidad predictiva aportado por la percepción de similaridad al prototipo; evidencian la contribución diferencial de autoeficacia y anticipación de dificultades a la predicción de la intención y conducta.
\end{abstract}

Palabras clave: Consumo de tabaco, autoeficacia, similaridad al prototipo, intención de conducta, adolescencia.

\section{Psychosocial predictors of adolescent smoking behavior: extensions to the Theory of Planned Behavior}

\begin{abstract}
This research examined, first, the incremental predictive utility of prototype similarity after variables from the theory of planned behavior (TPB) had been controlled, and, second, the differential predictive contribution of the two ingredients of the perceived behavioral control (PBC): selfefficacy belief and perceived difficulty. 227 adolescents, completed, along two months, measures of TPB variables, prototype similarity, and smoking behavior. Data analyses evidenced that: 1) Intention not to smoke and smoking behavior were predicted by TPB variables (except for subjective norm). 2) Prototype similarity significantly added to predictions of intention and behavior. 3) Both PBC ingredients were predictive of intention, but only self-efficacy was predictive of behavior. Overall, these findings: are globally consistent with TPB research; evidence the predictive advantage of the perception of prototype similarity, expanding in this way the predictive utility of the TPB; finally, show the differential contribution of self-efficacy and perceived difficulty in predicting intention and behavior.
\end{abstract}

Keywords: Smoking behavior, self-efficacy, prototype similarity, behavioral intention, adolescence.

\section{INTRODUCCIÓN}

Hoy es plenamente reconocido que una parte significativa de los problemas de salud que aquejan a la población guardan una estrecha relación con determinadas formas de conducta y estilos de vida. En este contexto, el consumo de tabaco constituye un ejemplo paradigmáti-

Recibido 6 mayo 2008; aceptado 11 junio 2008

Correspondencia: José Bermúdez, Departamento de Psicología de la Personalidad, Evaluación y Tratamiento Psicológicos, Facultad de Psicología, UNED, c/ Juan del Rosal, 10, 28040 Madrid. Correo-e: jbermudez@psi.uned.es co de lo que estamos comentando. De acuerdo con los datos aportados por la última Encuesta Nacional de Salud publicada (Ministerio de Sanidad y Consumo, 2004), en el año 2003 más de 60.000 personas han fallecido en España como consecuencia del consumo de tabaco, que ya es la principal causa de enfermedad, invalidez y muerte evitable. La elevada tasa de mortalidad y morbilidad asociada al consumo de tabaco, unida al hecho de que el consumo de tabaco es un factor de riesgo susceptible de prevención, han convertido la reducción de la prevalencia del consumo de tabaco en uno de los objetivos 
prioritarios de las políticas y actuaciones dirigidas a la mejora de los niveles de salud de la población. Abunda en esta necesidad de prevención la observación de que el consumo de tabaco se viene produciendo a edades cada vez más tempranas, situándose actualmente la edad de inicio en los adolescentes españoles en torno a los 12 años (Moreno, Muñoz, Pérez y Sánchez-Queija, 2005), con un consumo medio diario de 7,4 cigarrillos. Dato que resulta particularmente preocupante por cuanto es conocida la asociación entre el inicio temprano en el consumo y la intensidad y consolidación del hábito (Cortés, Schiaffino, Martí y Fernández, 2005; Higgins y Conner, 2003). Todo ello aboga por la necesidad de identificar aquellos factores que expliquen el inicio y mantenimiento de este tipo de conductas que, como el consumo de tabaco, suponen un riesgo cierto para la salud, lo que nos permitiría diseñar estrategias preventivas adecuadas para hacerles frente y cortocircuitar su incidencia.

Con este propósito general se han formulado una serie de modelos e hipótesis teóricos, al amparo del paraguas teórico constituido por el acercamiento socio-cognitivo-afectivo al estudio de la conducta (ver revisiones en Armitage y Conner, 2000; Bermúdez, 1999, 2006; Gebhardt y Maes, 2001; Maes y Karoly, 2005;). De entre estos modelos, el más ampliamente empleado ha sido la teoría de la conducta planificada (TCP, en adelante) (Ajzen, 1991), que viene a explicar entre un 30 y un $53 \%$ de la varianza de la intención de conducta y en torno al $40 \%$ de la varianza de la conducta en diversos estudios prospectivos (ver revisiones en Ajzen, 1991; Armitage y Conner, 2001; Godin y Kok, 1996; Randall y Wolff, 1994; Sheeran, 2002; Sheeran y Orbell, 1998; Sheeran y Taylor, 1999; Sutton, 1998; Trafimow, Sheeran, Conner, y Finlay, 2002).

En esencia, este acercamiento teórico sugiere que la intención, que representa la base motivacional que lleva al individuo a tomar la decisión de emprender un determinado curso de acción, es el determinante inmediato de la conducta, de cuya varianza viene a explicar entre el 20 y el 30\% (ver revisiones en Abraham, Sheeran y Johnston, 1998; Hausenblas, Carron y Mack, 1997). Este sustrato motivacional de la conducta es fruto, a su vez, de tres factores, actitud, norma subjetiva y control conductual per- cibido. La actitud recoge la valoración que el individuo hace de la conducta, en base, esencialmente, a la ponderación que establece entre los resultados que espera obtener con la conducta y el esfuerzo y coste que le puede suponer su logro («balance de resultados», en definitiva). Con el concepto de norma subjetiva se hace referencia a la percepción que tiene el individuo de la presión social a la que se enfrenta para que lleve a cabo, o no, la conducta. Estos dos componentes explican entre un 33 y $50 \%$ de la varianza de la intención (Armitage y Conner, 2001; Sheeran y Taylor, 1999). Por último, control conductual percibido refiere a la percepción de control sobre la conducta, a partir de la percepción de la disponibilidad de recursos personales apropiados y la expectativa de potenciales dificultades y obstáculos para llevar a cabo la conducta. Este componente se postula como determinante, tanto de la intención (aportaciones medias en torno al 11-13\% de la varianza; Godin y Kok, 1996; Trafimow et al., 2002) y, en la medida en que esta percepción de control refleja realmente el control que la persona tiene sobre la conducta, puede ser igualmente un determinante directo de la conducta (aportaciones medias en torno al 5-6\% de la varianza; ver metaanálisis de Armitage y Conner, 2001; Sheeran y Taylor, 1999).

Conjugando estos elementos, la predicción genérica que se hace desde la TCP sería que mientras más positiva es la actitud, se disponga de apoyo social (básicamente entre aquellos que sirven al individuo como referentes importantes) y se perciba control sobre la conducta, más probable será que el individuo tome la decisión de llevarla a cabo y, a su vez, mientras más potente sea esta decisión, la intención de realizar la conducta, mayor será la probabilidad de que, de hecho, se lleve a cabo.

Desde este marco de referencia se ha desarrollado un importante volumen de investigación, que ha puesto de manifiesto su utilidad para predecir la formulación de intención de conducta (el proceso de toma de decisión) y el desarrollo de la conducta consecuente con dicha intención, en un amplio rango de conductas, tales como participación en programas de cribado (screening), programas de actividad física regular, adherencia a los regímenes y prescripciones 
médicos, consumo de tabaco, alcohol, drogas, conducta sexual de riesgo, etc. (ver revisiones de Armitage y Conner, 2001; Godin y Kok, 1996; Maes y Karoly, 2005; Sheeran, 2002).

A modo de balance global, estas investigaciones apoyan sustancialmente las hipótesis formuladas por la TCP, tanto por lo que respecta al valor de la intención conductual como determinante próximo de la conducta, como por lo que concierne al papel de los distintos procesos y factores y la dinámica de relaciones existentes entre los mismos que contribuyen al desarrollo de la intención y, por tanto, su valor heurístico para el diseño de programas preventivos y promotores de salud.

Con todo, su potencial explicativo deja aún un amplio porcentaje de varianza por explicar, tanto por lo que respecta al proceso de toma de decisión, como al propio desarrollo de la conducta consistente con dicha decisión. Con el propósito de mejorar esta situación, se viene sugiriendo en los últimos años la conveniencia de, por una parte, explorar la contribución de otros factores que podrían incrementar la validez predictiva del modelo $\mathrm{y}$, por otra, profundizar en la naturaleza y utilidad predictiva diferencial de los distintos elementos que integran los principales constructos del modelo.

En el primer caso, aunque la investigación acumulada es aún más bien escasa, se está explorando la utilidad predictiva incremental de factores de influencia social como la norma descriptiva y la percepción de similaridad al prototipo de persona que desarrolla la conducta objeto de estudio (Rivis y Sheeran, 2003a,b; Rivis, Sheeran, y Armitage, 2006; Wilkinson y Abraham, 2004), la percepción de la conducta en términos morales (Godin, Conner, y Sheeran, 2005; McMillan, Higgins y Conner, 2005), o la anticipación de potenciales consecuencias emocionales negativas asociadas a un particular curso de acción (Conner, Sandberg, McMillan y Higgins, 2006). De estas variables, probablemente la de mayor significación para el estudio de la conducta adolescente sea la percepción de la conducta de los iguales y la percepción del grado en que uno se siente similar a la imagen prototípica de quien realiza la conducta, teniendo en cuenta el papel que en esta edad, en la que el individuo está conformando su identidad personal (Erikson, 1968), juegan estos factores de modelado e imitación social (Aloise-Young y Hennigan, 1996; Aloise-Young, Hennigan, y Graham, 1996; Barton, Chassin y Presson, 1982; Chassin, Presson, Sherman, Corty y Olshavsky, 1981). A este respecto, si bien existe evidencia empírica que apoya el papel facilitador que la valoración de las características del prototipo juega en la predicción de la conducta en la adolescencia (Blanton, van den Eijnden, Buunk, Gibbons, Gerrard y Bakker, 2001; Gibbons, Gerrard y Lane, 2003; Gibbons, Gerrard y McCoy, 1995; Spijkerman, van den Eijnden, Vitale y Engels, 2004; van den Eijnden, Spijkerman y Engels, 2006), es ciertamente escasa la investigación centrada en el análisis de la contribución relativa que la percepción de similaridad al prototipo puede tener para la predicción del proceso de decisión y desarrollo efectivo de la conducta (y de manera particular en el caso del consumo de tabaco), una vez controlado el efecto de otros factores sociocognitivos suficientemente consolidados, como sería el caso de los contemplados en la TCP.

Por lo que concierne al segundo frente de investigación, el grueso de la misma se ha centrado en el análisis de la utilidad predictiva diferencial de los componentes que integran el factor control conductual percibido, constructo en el que, ya desde la conceptualización original propuesta por Ajzen $(1985,1991)$, se amalgaman con frecuencia aspectos ligados a la anticipación de dificultades, con otros asociados a la percepción de control y valoración que hace el sujeto acerca de su capacidad y la disponibilidad de recursos para llevar a cabo la conducta (connotación esta última próxima al concepto de autoeficacia sugerido por Bandura, 1982,1986). Si bien en este caso la investigación es algo más extensa (prácticamente inexistente, no obstante, por lo que respecta a la explicación del consumo de tabaco en población adolescente), persiste, sin embargo, una notable ambigüedad en los resultados y aportaciones de la misma, en gran medida debido a la falta de claridad a la hora de evaluar los determinantes internos y externos que confluyen en la percepción de controlabilidad de la conducta. Así, la estrategia más frecuentemente em- 
pleada ha consistido en contrastar en qué medida la intención de llevar a cabo la conducta y su implementación efectiva vienen determinadas por el grado en que uno cree tener control sobre la conducta o por el grado en que uno estima que la ejecución de la conducta le resultará fácil o difícil (ver revisiones en Conner y Armitage, 1998; Povey, Conner, Sparks, James y Shepherd, 2000; Trafimow, et al., 2002). En ambos casos, sin embargo, la apreciación del sujeto puede venir condicionada por factores, tanto internos (capacidad, motivación, experiencia previa, etc.), como externos (oportunidad, requerimientos específicos de la conducta, etc.); en consecuencia, atribuir la determinación de la intención y conducta a una razón (percepción de control) u otra (percepción de dificultad) puede no aclararnos las razones efectivas de la misma. Para dar respuesta a esta problemática, en nuestra investigación se diferencian la percepción de control originada en la confianza y seguridad en que se poseen la capacidad y recursos para llevar a cabo la conducta (emplearemos en este caso el constructo autoeficacia) y la anticipación de dificultades, como criterio externo de controlabilidad de la conducta.

Resumiendo, en este contexto, mediante un diseño prospectivo, nuestra investigación se ha proyectado con un doble objetivo, por un lado, estudiar la utilidad predictiva incremental de la percepción de similaridad al prototipo de fumador, una vez controlado el efecto de los otros factores contemplados en la TCP, y, por otro, analizar la contribución diferencial de los dos componentes del control conductual percibido (anticipación de posibles dificultades y percepción de autoeficacia), a partir de la evaluación netamente diferenciada de ambos.

\section{MÉTODO}

\section{Muestra}

La muestra estuvo formada por 227 estudiantes de secundaria, 96 varones y 131 mujeres. Edad media $=15-18$ años $(D T=1.25$, rango: 13-17), que participaron voluntariamente en la investigación.

\section{Procedimiento}

En una primera evaluación, los sujetos contestaron a un cuestionario conteniendo items relativos a la conducta relacionada con el consumo de tabaco, así como de las variables que potencialmente contribuirían a este tipo de conducta entre los adolescentes. Posteriormente, a los dos meses se evaluó el cumplimiento de la intención conductual.

\section{Variables e Instrumentos de medida}

Específicamente para esta investigación se elaboraron una serie de escalas para medir las diferentes variables de interés. Todos los items, salvo cuando expresamente se indica lo contrario, fueron respondidos en escalas tipo Likert de 10 puntos, ancladas verbalmente en cada extremo:

Historia de consumo: se evaluó pidiendo al sujeto indicara la alternativa que correspondía a su situación personal con relación al consumo de tabaco: $1=$ Nunca he fumado, ni siquiera un cigarrillo; $2=\mathrm{He}$ probado alguna vez, pero no más de 5 cigarrillos en total; $3=$ Fumo de vez en cuando; 4= Fumo regularmente, al menos una vez a la semana; $5=$ Fumo todos los días; $6=\mathrm{He}$ dejado de fumar (hace menos de 1 mes; hace entre 2 y 6 meses; Hace más de 6 meses). Similaridad al prototipo de fumador: se evaluó con el ítem «¿En qué medida crees que te pareces al fumador típico?» Nada en absoluto Plenamente. Presión normativa: medida mediante el ítem «¿Cómo crees que aquellas personas que son importantes para ti (padres, amigos, otras) valorarían el que no fumases (o continúes sin fumar)?» Negativamente - Positivamente. Motivación para acceder a la presión: evaluada en respuesta a la pregunta, «Con relación a tu intención de dejar de fumar (o continuar sin fumar), ¿en qué medida te importa (y procurarías hacer caso a) la opinión de las personas que son importantes en tu vida?» Nada Mucho. Actitud: 7 escalas de adjetivo bipolares, en respuesta a la pregunta «¿En qué medida crees que intentar dejar de fumar (o continuar sin fumar) es, o puede ser ...?» (inútil-útil; estresante-relajante,...) $(\mathrm{alfa}=0,85)$. Percepción de 
Autoeficacia: 12 items evaluaron el grado en que uno se siente capaz de no fumar en distintas situaciones (p.ej.: «aunque te sientas solo y deprimido», «estés en una fiesta con amigos»,...), No estoy en absoluto seguro de que lo pudiera lograr - Estoy completamente seguro de que lo podría lograr, (alfa $=0,96)$. Anticipación de Dificultades: se midió mediante el ítem, «¿Crees que encontrarías dificultades para dejar de fumar (o continuar sin fumar) si te lo propusieras?» Ninguna - Muchas. Decisión: un ítem preguntaba por la intención de dejar de fumar (o continuar sin fumar), «¿Estás dispuesto a dejar de fumar (o continuar sin fumar)?» No, en absoluto - Sí, definitivamente. Fuerza de la Decisión: se evaluó mediante el ítem, «Si realmente estás decidido a dejar de fumar (o continuar sin fumar), ¿qué seguridad tienes en que lograrás mantener esta decisión?» Muy poca - Total. Conducta: en la evaluación de seguimiento se midió el grado en que el sujeto había cumplido con la decisión tomada, mediante el siguiente ítem «Desde que contestaste al anterior cuestionario, marca la alternativa que corresponda a tu situación personal»: 1 (He comenzado a fumar; Sigo fumando; He aumentado el consumo de tabaco; Había dejado de fumar, pero he vuelto a fumar de nuevo), 2 (He disminuido el consumo de tabaco; He intentado dejar de fumar, pero no lo he conseguido); 3 (He continuado sin fumar; He dejado de fumar).

Con base en estas escalas se computaron las siguientes variables compuestas: Norma subjetiva (Presión Normativa $\times$ Motivación para ac- ceder a la presión); Intención (Decisión $\times$ Fuerza de la Decisión), Control Conductual Percibido (Percepción de Autoeficacia - Anticipación de Dificultades), que, junto a las medidas evaluadas directamente (Actitud, Similaridad al prototipo de fumador y Conducta), constituyen las variables relevantes para esta investigación.

\section{RESULTADOS}

Para dar respuesta a los objetivos de investigación, se llevaron a cabo una serie análisis de regresión jerárquica.

\section{Estadísticos descriptivos y correlaciones entre las variables}

Los datos descriptivos indican que la muestra tenía una bastante favorable actitud, norma subjetiva, percepción de control conductual, autoeficacia, intención e implementación conductual, y, por otro lado, una baja percepción de similaridad al prototipo de fumador y bajas expectativas de dificultad. Este cuadro global positivo podría ser debido a la presencia de una mayor proporción de no-fumadores y exfumadores en la muestra $(69,16 \%)$.

Las correlaciones, por su parte, entre las variables de la TCP, por una parte, y los niveles de intención y conducta, por otra, fueron en general moderadas y significativas (excepto para la relación entre norma subjetiva y conducta), como era de esperar (Tabla 1).

Tabla 1. Estadísticos descriptivos [Media (DT)] e intercorrelaciones entre las variables del estudio

\begin{tabular}{|c|c|c|c|c|c|c|c|c|}
\hline \multirow{2}{*}{\multicolumn{9}{|c|}{ 1. Similaridad }} \\
\hline & & & & & & & & \\
\hline 2. Actitud & $-0,43 * * *$ & & & & & & & \\
\hline 3. Norma subjetiva & $-0,15^{*}$ & $0,29 * * *$ & & & & & & \\
\hline 4. Control percibido & $-0,43 * * *$ & $0,45^{* * *}$ & $0,22 * * *$ & & & & & \\
\hline 5. Dificultad percibida & $0,42 * * *$ & $-0,29 * * *$ & $-0,15^{*}$ & $-0,54 * * *$ & & & & \\
\hline 6. Autoeficacia & $-0,41 * * *$ & $0,45^{* * *}$ & $0,22 * * *$ & $0,82 * * *$ & $-0,46 * * *$ & & & \\
\hline 7. Intención & $-0,58 * * *$ & $0,45 * * *$ & $0,24 * * *$ & $0,57 * * *$ & $-0,50 * * *$ & $0,55^{* * *}$ & & \\
\hline 8. Conducta & $-0,44 * * *$ & $0,46 * * *$ & 0,17 & $0,53 * * *$ & $-0,27 * *$ & $0,52 * * *$ & $0,60 * * *$ & \\
\hline Media & 3,69 & 57,62 & 72,93 & 79,47 & 4,11 & 83,59 & 48,70 & 2,54 \\
\hline$D T$ & 2,33 & 10,89 & 27,55 & 31,77 & 3,16 & 30,18 & 24,92 & 0,81 \\
\hline
\end{tabular}

Nota: $N=227$ (excepto Conducta, donde $N=106$ ). ${ }^{*} p<0,05 ; * * p<0,01 ; * * * p<0,001$. 


\section{Procesos psicológicos explicativos} de la intención de no fumar o dejar de fumar y la implementación conductual de la intención

Los resultados del modelo global, tomando todos los sujetos, muestran que la intención de no fumar o dejar de fumar es explicada positivamente por la percepción de control sobre la conducta y actitud y negativamente por la percepción de similaridad al prototipo fumador (Tabla 2).

En la primera ecuación, las variables de la TCP explicaron el $38 \%$ de la varianza en intención, debido a la significativa contribución de la actitud y la percepción de control conductual. La adición en la segunda ecuación de la percepción de similaridad al prototipo de fumador, supuso un incremento significativo de un $10 \%$ en la varianza explicada de la intención de conducta $\left(\Delta R^{2}=0,10 ; \Delta F=42,12\right.$, $p<0,001)$.

Por otra parte, por lo que concierne al cumplimiento de la intención, los resultados revelaron que el mismo viene explicado negativamente por la percepción de similaridad al prototipo de fumador y positivamente por la intención y la percepción de control sobre la conducta (Tabla 3).

En la primera ecuación, las variables de la TCP explicaron el $44 \%$ de la varianza en conducta, debido a la significativa contribución de la intención y la percepción de control sobre la conducta. La adición en la segunda ecuación de la percepción de similaridad al prototipo fumador, supuso un incremento significativo de un $2 \%$ en la varianza explicada de la conducta $\left(\Delta R^{2}=0,02 ; \Delta F=3,93, p<0,05\right)$.

Tabla 2. Análisis de regresión jerárquica.

Predicción de la intención de conducta a partir de las variables TCP $(N=227)$

\begin{tabular}{llcc}
\hline Paso & Variables & $\beta$ & $\beta$ \\
\hline 1 & Control percibido & $0,45^{* * *}$ & $0,34^{* * *}$ \\
& Actitud & $0,23^{* * *}$ & $0,12^{*}$ \\
& Norma subjetiva & 0,08 & 0,08 \\
& Similaridad & & $-0,37^{* * *}$ \\
2 & & & 0,48 \\
$R^{2}$ & & 0,38 & $50,20^{* * *}$ \\
$F$ & & $44,63^{* * *}$ & \\
\hline
\end{tabular}

Nota: $* p<0,05 ; * * * p<0,001$.

Tabla 3. Análisis de regresión jerárquica.

Predicción de la conducta a partir de las variables TCP $(N=106)$

\begin{tabular}{llcc}
\hline Paso & Variables & $\beta$ & $\beta$ \\
\hline 1 & Control percibido & $0,25^{* * *}$ & $0,24^{* * *}$ \\
& Actitud & 0,14 & 0,12 \\
& Norma subjetiva & 0,06 & 0,07 \\
& Intención & $0,40^{* * *}$ & $0,33^{* * *}$ \\
& Similaridad & & $-0,17^{*}$ \\
2 & & & 0,46 \\
$R^{2}$ & & 0,44 & $17,31^{* * *}$ \\
$F$ & & $20,06^{* * *}$ &
\end{tabular}

Nota: $* p<0,05 ; * * p<0,01 ; * * * p<0,001$. 
Tabla 4. Análisis de regresión jerárquica.

Utilidad predictiva diferencial de la anticipación de Dificultad

y percepción de Autoeficacia, sobre la intención de conducta $(N=227)$.

\begin{tabular}{|c|c|c|c|c|c|c|}
\hline Paso & Variables & $\beta$ & $R^{2}$ & $F$ & $\Delta \mathrm{R}^{2}$ & $\Delta F$ \\
\hline 1 & $\begin{array}{l}\text { Actitud } \\
\text { Similaridad }\end{array}$ & $\begin{array}{r}0,25^{* * *} \\
-0,47^{* * *}\end{array}$ & 0,38 & $69,58 * * *$ & 0,38 & $69,58 * * *$ \\
\hline $2 a$ & $\begin{array}{l}\text { Actitud } \\
\text { Similaridad } \\
\text { Dificultad }\end{array}$ & $\begin{array}{r}0,21 * * * \\
-0,37 * * * \\
-0,28 * * *\end{array}$ & 0,45 & $60,13 * * *$ & 0,07 & $27,78^{* * *}$ \\
\hline $2 b$ & $\begin{array}{l}\text { Actitud } \\
\text { Similaridad } \\
\text { Autoeficacia }\end{array}$ & $\begin{array}{c}0,15^{*} \\
-0,38^{* * *} \\
0,32^{* * *}\end{array}$ & 0,46 & $63,51 * * *$ & 0,08 & $32,01 * * *$ \\
\hline 3 & $\begin{array}{l}\text { Actitud } \\
\text { Similaridad } \\
\text { Dificultad } \\
\text { Autoeficacia }\end{array}$ & $\begin{array}{c}0,14 * \\
-0,33 * * * \\
-0,20 * * * \\
0,25 * * *\end{array}$ & 0,49 & $53,40^{* * *}$ & a & a \\
\hline
\end{tabular}

Notas: $* p<0,05 ; * * * p<0,001$.

${ }^{a}$ La introducción de la percepción de Autoeficacia tras la anticipación de Dificultad (pasos 2a-3), incrementó significativamente $R^{2}\left(\Delta R^{2}=0,04 ; \Delta F=18,76, p<0,001\right)$; la adición de la anticipación de Dificultad tras la percepción de Autoeficacia (pasos 2b-3) también produjo incremento significativo en $R^{2}\left(\Delta R^{2}=0,03 ; \Delta F=12,88, p<0,001\right)$.

\section{Papel diferencial de los componentes de la percepción de control conductual}

Como se recoge en la Tabla 4, la intención de no fumar o dejar de fumar es explicada, junto a actitud y percepción de similaridad al prototipo de fumador (que explican el 38\% de la varianza), por la anticipación de dificultades para llevar a cabo la conducta (negativamente) y por la percepción de autoeficacia (positivamente), que añaden un $7 \%$ y $8 \%$, respectivamente, de la varianza explicada. Los análisis de la aportación diferencial de cada componente de la percepción de control conductual, muestran una contribución prácticamente similar de ambos, un $4 \%$ en el caso de la percepción de autoeficacia, una vez controlado el efecto de actitud, percepción de similaridad al prototipo y anticipación de dificultades (contraste entre los pasos 2a-3), y un 3\% en el caso de la anticipación de dificultades, una vez controlado el efecto de actitud, percepción de similaridad al prototipo y percepción de autoeficacia (contraste entre los pasos $2 b-3$ ).
Por lo que respecta a la implementación conductual de la intención, junto a la contribución explicativa de la intención conductual y la percepción de similaridad al prototipo que explican el 39\% de la varianza), se aprecia, en cambio, una significativa diferenciación en la contribución de los dos componentes de la percepción de control conductual, mostrando los resultados un mayor peso explicativo de la percepción de autoeficacia $\left(\Delta R^{2}=0,06\right.$; $p<0,01$ ), frente a la prácticamente nula contribución de la percepción de dificultades $\left(\Delta R^{2}\right.$ $=0,01 ;$ n.s. $)$ (Tabla 5). Consecuentemente, los análisis de la contribución diferencial de cada componente de la percepción de control conductual (contrastes 2a-3 y 2b-3, para percepción de autoeficacia y anticipación de dificultades, respectivamente), mostraron que sólo la percepción de autoeficacia hacía una contribución adicional significativa, incrementando la varianza explicada en un $5 \%$, una vez controlado el efecto de la intención, percepción de similaridad al prototipo y anticipación de dificultades. 
Tabla 5. Análisis de regresión jerárquica. Utilidad predictiva difeencial de la anticipación de Dificultad y percepción de autoeficacia, sobre la conducta $(N=106)$

\begin{tabular}{|c|c|c|c|c|c|c|}
\hline Paso & Variables & $\beta$ & $R^{2}$ & $F$ & $\Delta \mathrm{R}^{2}$ & $\Delta F$ \\
\hline 1 & $\begin{array}{l}\text { Similaridad } \\
\text { Intención }\end{array}$ & $\begin{array}{l}-0,21 * \\
0,50 * * *\end{array}$ & 0,39 & $32,83 * * *$ & 0,39 & $32,83 * * *$ \\
\hline $2 a$ & $\begin{array}{l}\text { Similaridad } \\
\text { Intención } \\
\text { Dificultad }\end{array}$ & $\begin{array}{l}-0,20^{*} \\
0,48^{* * *} \\
-0,08\end{array}$ & 0,40 & $22,23 * * *$ & 0,01 & 1,01 \\
\hline $2 b$ & $\begin{array}{l}\text { Similaridad } \\
\text { Intención } \\
\text { Autoeficacia }\end{array}$ & $\begin{array}{c}-0,18^{*} \\
0,37 * * * \\
0,27 * *\end{array}$ & 0,45 & $27,31 * * *$ & 0,06 & $10,29 * *$ \\
\hline 3 & $\begin{array}{l}\text { Similaridad } \\
\text { Intención } \\
\text { Dificultad } \\
\text { Autoeficacia }\end{array}$ & $\begin{array}{l}-0,18^{*} \\
0,37 * * * \\
-0,03 \\
0,27 * *\end{array}$ & 0,45 & $20,36^{* * *} *$ & a & $\mathrm{a}$ \\
\hline
\end{tabular}

Nota: $* p<0,05 ; * * p<0,01 ; * * * p<0,001$

${ }^{a}$ La introducción de la percepción de Autoeficacia tras la anticipación de Dificultad (pasos 2a-3), incrementó significativamente $R^{2}\left(\Delta \mathrm{R}^{2}=0,05 ; \Delta F=9,28, p<0,01\right)$; mientras que la adición de la anticipación de Dificultad tras la percepción de Autoeficacia (pasos 2b-3), no produjo incremento significativo alguno en $R^{2}\left(\Delta R^{2}=0,001 ; \Delta F=0,17, n\right.$.s.).

la percepción de autoeficacia hacía una contribución adicional significativa, incrementando la varianza explicada en un $5 \%$, una vez controlado el efecto de la intención, percepción de similaridad al prototipo y anticipación de dificultades.

\section{DISCUSIÓN Y CONCLUSIONES}

Nuestra investigación tenía un doble objetivo; por una parte, estudiar la utilidad predictiva incremental de la percepción de similaridad al prototipo de fumador y, por otra, analizar la contribución diferencial de los dos componentes del control conductual percibido.

Los resultados, en general, prestan apoyo a las hipótesis de la TCP, al mostrar cómo la intención de no fumar o dejar de fumar depende esencialmente del control conductual percibido y de la actitud hacia la conducta; mientras el cumplimiento efectivo de dicha intención está asociado significativamente a la fuerza motivacional expresada en la intención junto a la percepción de control sobre la conducta que uno se ha propuesto llevar a cabo. Menor relevancia parece tener, sin embargo, la percepción de presión normativa, tanto a la hora de formar la intención de conducta, cuanto sobre la implementación efectiva de la misma. Esta menor relevancia explicativa de los factores de influencia social recogidos en el constructo «Norma Subjetiva», no se desvía, sin embargo, de la investigación derivada de la TCP, como se desprende de los estudios metaanalíticos que vienen a poner de manifiesto que el componente Norma Subjetiva suele ser el predictor más débil, tanto de la intención como de la implementación conductual, en comparación con la Actitud o el Control Conductual Percibido (Armitage y Conner, 2001; Godin y Kok, 1996; Hausenblas et al., 1997; Sheeran y Orbell, 1998). Evidencia, por otra parte, sobre la que se apoya investigación previa y que motiva el primer objetivo de nuestra investigación, al abogar por la necesidad de explorar la potencial utilidad predictiva de otros factores de influencia social que no tengan una carga normativa tan decisiva [al enfatizar la presión social percibida para llevar a cabo, o no, una determinada conducta] 
significativa contribución que la percepción de Similaridad al prototipo de fumador presta a la hora de predecir, tanto la intención como el desarrollo de la conducta intencional, al incrementar el porcentaje de varianza explicada en un $10 \%$ y un $2 \%$ para intención y conducta, respectivamente, una vez controlado el efecto de las variables tradicionalmente contempladas en la TCP. El percibirse próximo a la imagen prototípica que uno tiene de la persona que fuma, actuaría como un factor que dificultaría el tomar la decisión de abandonar el consumo de tabaco y mantener la abstinencia y el desarrollo de la conducta consecuente. Estos resultados son plenamente consistentes con investigaciones previas (Moan y Rise, 2006; Spijkerman, et al., 2004; Rivis et al., 2003b, 2006) y abogan por la conveniencia de extender el rango de factores predictivos contemplados en la TCP, para acoger este tipo de factores de influencia social que, al menos en población adolescente, como ya hemos comentado, parece revestir una mayor significación explicativa que otros tal vez más restrictivos, como sería el caso del componente tradicionalmente contemplado en la variable Norma Subjetiva.

El papel de la percepción de similaridad sobre la intención de conducta podía esperarse, habida cuenta el papel que en la TCP se atribuye a los factores de influencia social en la determinación del nivel motivacional adecuado para que el sujeto tome la decisión conductual correspondiente. Su acción directa sobre la conducta resulta, en cambio, algo más sorpresiva desde los supuestos teóricos de la TCP; un resultado semejante se obtuvo en la investigación de Rivis et al., 2003b, si bien referido al desarrollo de actividad física regular. Dada la naturaleza correlacional de nuestra investigación, no disponemos, sin embargo, de argumentos sólidos para dar cuenta de este resultado y, por ello, tan sólo tentativamente podrían aventurarse interrogantes acerca de potenciales mecanismos explicativos, a los que merecería prestar atención específica en futuras investigaciones. Así, podría ser que la percepción de similaridad al tipo de persona que desarrolla la conducta en estudio actúe como reforzador directo de la forma de conducta consistente con la que desarrolla el tipo de persona con la que uno se identifica; aplicado a nuestro estudio, ello supondría que el percibirse similar al prototipo de fumador actuaría como reforzador del consumo, dificultando, por tanto, el cambio propuesto de abandonar el consumo y mantener la abstinencia; el cambio de conducta sería, por su parte, más fácil, a medida que disminuye el grado de similaridad con el prototipo fumador. Bien podría ser, por otra parte, que este efecto sobre la conducta fuese indirecto, mediado por la percepción de apoyo social generado al percibirse integrado en la red de personas que comparten las mismas características y formas de conducta del prototipo, cuya imagen se toma como modelo desde la que uno construye su propia identidad personal. En todo caso, éstas no pasan de ser conjeturas necesitadas de investigación futura.

Por lo que concierne al segundo objetivo de nuestra investigación, los resultados obtenidos apoyan la contribución significativa al desarrollo de la intención conductual de ambos componentes de la percepción de control conductual; esto es, la anticipación de posibles dificultades para llevar a cabo la futura conducta influye negativamente a la hora de tomar la decisión de abandonar el consumo de tabaco y mantenerse abstinente, mientras el percibirse con recursos suficientes para asumir tal compromiso facilitaría la toma de decisión. Sin embargo, a la hora de explicar el desarrollo efectivo de la conducta comprometida, los resultados de nuestro estudio muestran una contribución netamente diferenciada de ambos componentes de la percepción de control conductual. Así, mientras desaparece la relevancia que en el momento de tomar la decisión tenía la anticipación de dificultades, una vez se ha tomado la decisión, la conducta se ve facilitada significativamente por la percepción de recursos para hacer frente a los posibles obstáculos que uno pueda encontrar para llevar a cabo la intención de conducta.

Estos resultados, en consonancia con la investigación previa sobre el tema, evidencian la contribución específica de estos dos componentes de la percepción de control sobre la conducta, así como su papel diferencial en distintas fases del desarrollo de la conducta (proceso de decisión e implementación efectiva de la con- 
ducta) (Conner et al., 1998; Povey et al., 2000; Trafimow et al., 2002). La aparente divergencia entre nuestros resultados, que muestran una mayor relevancia explicativa de la percepción de autoeficacia, tanto sobre la intención conductual, como sobre la conducta misma, y aquellos en los que se sostiene la prevalencia explicativa de la percepción de dificultad, se debería esencialmente a la distinta operacionalización que en un caso y otro se hace de los componentes de la percepción de control conductual. Así, cuando en los estudios revisados en el metaanálisis llevado a cabo por Trafimow et al. (2002), se habla de dificultad percibida, se está evaluando en realidad una de las facetas contempladas en la conceptualización original de la percepción de autoeficacia, esto es, el grado en que el sujeto estima que la conducta le resultará fácil o difícil; de hecho, en muchos de estos estudios se da el nombre de autoeficacia a este componente de la percepción de control conductual (Manstead y van Eekelen, 1998; Povey et al., 2000; Terry y O'Leary, 1995; White, Terry y Hogg, 1994). En nuestra investigación, en cambio, la valoración de la facilidad o dificultad para llevar a cabo la conducta estaría subsumida en la valoración que hace el sujeto al evaluar su capacidad para realizar la conducta, considerando un espectro amplio de situaciones que podrían dificultar su ejecución; hablamos en este caso de percepción de autoeficacia en sentido propio. Frente a esta fuente interna de control, la estimación de posibles dificultades a las que uno podría enfrentarse para llevar a cabo la conducta, nos permite apreciar el impacto de factores externos de control relativamente independientes a los contemplados en la percepción de autoeficacia.

La prevalencia explicativa que muestran nuestros resultados a favor de la percepción de autoeficacia sería, por otra parte, un resultado genuino, no atribuible a posibles sesgos asociados al hecho del mayor número de items empleados para su evaluación. De hecho, análisis complementarios efectuados, tomando como medida de autoeficacia únicamente la puntuación en el ítem más representativo de la escala, arroja un perfil de resultados prácticamente similar por lo que concierne a su contribución diferencial específica, una vez controlado el efecto de las otras variables $\left(\Delta R^{2}=0,04 ; \Delta F=17,15, p<\right.$ 0,$001 ; \Delta R^{2}=0,07 ; \Delta F=14,08, p<0,001$, para intención y conducta, respectivamente). La contribución diferencial de ambos componentes a lo largo de las distintas fases del proceso de desarrollo de la conducta vendría en apoyo, por otra parte, de la investigación e hipótesis que vienen a sugerir el carácter esencialmente motivacional de los procesos que dominan la toma de decisión, mientras que el desarrollo de la conducta estaría gobernado en mayor medida por procesos volitivos y autorreguladores (Bandura, 1998, 2001; Bermúdez, 2006; Gollwitzer, 1990; Orbell y Sheeran, 2000). Desde esta perspectiva, la similar contribución de ambos componentes al desarrollo de la intención de conducta sugiere el valor adaptativo que para la adopción de una correcta decisión tiene la consideración y valoración, tanto de las potenciales dificultades y obstáculos, como de los recursos con los que se puede contar para llevar cabo la decisión que se está sopesando tomar. Una vez tomada la decisión, en cambio, lo importante es mantener la fuerza de la intención y el esfuerzo por alcanzar la meta, objetivo al que contribuirá la inatención o devaluación de potenciales dificultades y la focalización en la meta proyectada.

Somos conscientes de que este estudio adolece de ciertas limitaciones, que convendría tomar en consideración en posteriores investigaciones. Así, en primer lugar, la medida de conducta se ha basado en el autoinforme del sujeto, por lo que puede contener algún sesgo debido, entre otros, a la varianza que teóricamente comparte con la medición mediante cuestionario de las variables predictoras. Sería muy conveniente, por tanto, tomar indicadores directos de conducta y no confiar sólo en datos autoinformados. En segundo lugar, parece muy conveniente, al menos en este tipo de conductas, tomar periodos de seguimiento más amplios, que posibiliten la observación de cambios fiables en la conducta. En tercer lugar, si bien el número de items empleados para la evaluación de una variable, no necesariamente ha de condicionar significativamente el comportamiento de dicha variable, como hemos visto en el caso de la percepción de autoeficacia, no deja de ser recomendable, para asegurar la estabili- 
dad de la medida, basar la evaluación en más de un ítem. Por último, teniendo en cuenta la escasa existencia de investigación sobre la problemática abordada en nuestro estudio en su aplicación al consumo de tabaco en población adolescente, nuestros resultados deben ser tomados con prudencia en tanto nuevas investigaciones aportan mayor evidencia sobre el tema.

Pese a estas potenciales limitaciones, en el ámbito aplicado, finalmente, la contribución explicativa de la percepción de similaridad al prototipo, junto a la significación diferencial de los distintos aspectos implicados en la percepción de controlabilidad sobre la conducta, puestos de manifiesto en nuestra investigación, permitirían orientar mejor la evaluación hacia los factores sociocognitivos con mayor significación predictiva, así como el diseño de programas de intervención más apropiados en cada caso.

\section{REFERENCIAS}

Abraham, C., Sheeran, P., y Johnston, M. (1998). From health beliefs to self-regulation: Theoretical advances in the psychology of action control. Psychology and Health, 13, 569-592.

Ajzen, I. (1985). From intentions to actions: a theory of planned behavior. En J. Kuhl y J. Beckman (Eds.), Action-control: From cognition to behavior (pp. 11-39). Heidelberg: Springer.

Ajzen, I. (1991). The Theory of Planned Behavior. Organizational Behavior and Human Decision Processes, 50, 179-211.

Aloise-Young, P.A., y Hennigan, K.M. (1996). Self-image, the smoker stereotype and cigarette smoking: Developmental patterns. Journal of Adolescence, 19, 163-177.

Aloise-Young, P.A., Hennigan, K.M., y Graham, J.W. (1996). Role of the Self-Image and Smoker Stereotype in Smoking Onset during early Adolescence: A longitudinal study. Health Psychology, 15, 494-497.

Armitage, C.J., y Conner, M. (2000). Social cognition models and health behaviour: A structured review. Psychology and Health, 15, 173-189.

Armitage, C.J., y Conner, M. (2001). Efficacy of the Theory of Planned Behaviour: A meta-analytic review. British Journal of Social Psychology, 40, 471-499.

Bandura, A. (1982). Self-efficacy mechanism in human agency. American Psychologist, 37, 122-147.

Bandura, A. (1986). Social foundations of thought and action: a social-cognitive theory. Englewood Cliffs, NJ: Prentice-Hall.
Bandura, A. (1998). Health promotion from the perspective of Social Cognitive Theory. Psychology and Health, 13, 623-649.

Bandura, A. (2001). Social cognitive theory: An agentic perspective. Annual Review of Psychology, 52, 1-26.

Barton, J., Chassin, L., y Presson, C.C. (1982). Social image factors as motivators of smoking initiation in early and middle adolescence. Child Development, 53, 14991511.

Bermúdez, J. (1999). Personality and Health-Protective Behaviour. European Journal of Personality, 13, 83-103.

Bermúdez, J. (2006). Personality science, Self-Regulation, and Health Behavior. Applied Psychology: An International Review, 55, 386-396.

Blanton, H., van den Eijnden, R.J.J.M., Buunk, B.P., Gibbons, F.X., Gerrard, M., y Bakker, A. (2001). Accentuate the negative: Social images in the prediction and promotion of condom use. Journal of Applied Social Psychology, 31, 274-295.

Chassin, L., Presson, C.C., Sherman, S.J., Corty, E., y Olshavsky, R.W. (1981). Self-Images and Cigarette Smoking in Adolescence. Personality and Social Psychology Bulletin, 7, 670-676.

Conner, M., y Armitage, C.J. (1998). The theory of planned behaviour: A review and avenues for future research. Journal of Applied Social Psychology, 28, 14291463.

Conner, M., Sandberg, T., McMillan, B., y Higgins, A. (2006). Role of anticipated regret, intentions and intention stability in adolescent smoking initiation. British Journal of Health Psychology, 11, 85-101.

Cortés, M., Schiaffino, A., Martí, M., y Fernández, E. (2005). Factores cognitivos asociados con el inicio del consumo de tabaco en adolescentes. Gaceta Sanitaria, $19,36-44$.

Erikson, E.H. (1968). Identity: Youth and crisis. New York: Norton.

Gebhardt, W., y Maes, S. (2001). Integrating social-psychological frameworks for health behavior research. Psychology and Health, 13, 651-686.

Gibbons, F.X., Gerrard, M., y Lane, D.J. (2003). A socialreaction model of adolescent health risk. En J.M. Suls y K.A. Wallston (Eds.), Social psychological foundations of health and illness (pp. 107-136). Oxford: Blackwell.

Gibbons, F.X., Gerrard, M., y McCoy, S.B. (1995). Prototype perception predicts (lack of) pregnancy prevention. Personality and Social Psychology Bulletin, 21, 8593.

Godin, G., Conner, M., y Sheeran, P. (2005). Bridging the intention-behaviour 'gap': The role of moral norm. British Journal of Social Psychology, 44, 497-512.

Godin, G., y Kok, G. (1996). The Theory of Planned Behavior: A review of its applications to health-related 
behaviors. American Journal of Health Promotion, 11, 87-98.

Gollwitzer, P.M. (1990). Action phases and mind-sets. En E.T. Higgins y R.M. Sorrentino (Eds.), Handbook of motivation and cognition: Foundations of Social behavior (pp. 53-92). New York: Guilford.

Hausenblas, H.A., Carron, A.V., y Mack, D.E. (1997). Application of the theory of reasoned action and planned behavior to exercise behavior: A meta-analysis. Journal of Sport and Exercise Psychology, 19, 36-51.

Higgins, A., y Conner, M. (2003). Understanding adolescent smoking: the role of the Theory of Planned Behaviour and implementation intentions. Psychology, Health \& Medicine, 8, 173-186.

Maes, S., y Karoly, P. (2005). Self-Regulation Assessment and Intervention in Physical Health and Illness: A review. Applied Psychology: An International Review, 54, 267-299.

Manstead, A.S.R., y van Eekelen, S.A.M. (1998). Distinguishing between perceived control and self-efficacy in the domain of academic achievement and behavior. Journal of Applied Social Psychology, 28, 1375-1392.

McMillan, B., Higgins, A., y Conner, M. (2005). Using an extended theory of planned behaviour to understand smoking amongst schoolchildren. Addiction Research and Theory, 13, 293-306.

Ministerio de Sanidad y Consumo (2004). Encuesta Nacional de Salud de España 2003. Madrid: Ministerio de Sanidad y Consumo.

Moan, I.S., y Rise, J. (2006). Predicting smoking reduction among adolescents using an extended version of the theory of planned behaviour. Psychology \& Health, 21, 717-738.

Moreno, M.C., Muñoz, M.V., Pérez, P., y Sánchez-Queija, J. (2005). Los adolescentes españoles y su salud. Resumen del estudio «Health Behaviour in school aged children (HBSC)». Madrid: Ministerio de Sanidad y Consumo.

Orbell, S., y Sheeran, P. (2000). Motivational and volitional processes in action initiation: A field study of the role of implementation intentions. Journal of Applied Social Psychology, 30, 780-797.

Povey, R., Conner, M., Sparks, P., James, R., y Shepherd, R. (2000). Application of the Theory of Planned Behaviour to two dietary behaviours: Roles of perceived control and self-efficacy. British Journal of Health Psychology, 5, 121-139.

Randall, D.M., y Wolff, J.A. (1994). The time interval in the intention-behaviour relationship: Meta-analysis. British Journal of Social Psychology, 33, 405-418.

Rivis, A., y Sheeran, P. (2003a). Descriptive norms as an additional predictor in the Theory of Planned Behaviour: A Meta-Analysis. Current Psychology: Developmental, Learning, Personality, Social, 22, 218-233.
Rivis, A., y Sheeran, P. (2003b). Social influences and the Theory of Planned Behaviour: Evidence for a direct relationship between prototypes and young people's exercise behaviour. Psychology and Health, 18, 567583.

Rivis, A., Sheeran, P., y Armitage, C.J. (2006). Augmenting the theory of planned behaviour with the prototype/willingness model: Predictive validity of actor versus abstained prototypes for adolescents' health-protective and health-risk intentions. British Journal of Health Psychology, 11, 483-500.

Sheeran, P. (2002). Intention-Behavior relations: A conceptual and empirical review. European Review of Social Psychology, 12, 1-36.

Sheeran, P., y Orbell, S. (1998). Do intentions predict condom use? Meta-analysis and examination of six moderator variables. British Journal of Social Psychology, $37,231-250$.

Sheeran, P., y Taylor, S. (1999). Predicting intentions to use condoms: Meta-analysis and comparison of the theories of reasoned action and planned behavior. Journal of Applied Social Psychology, 29, 1624-1675.

Spijkerman, R., van den Eijnden, R.J.J.M., Vitale, S., y Engels, R.C.M.E. (2004). Explaining adolescents' smoking and drinking behavior: The concept of smoker and drinker prototypes in relation to variables of the theory of planned behavior. Addictive Behaviors, 29, 16151622.

Sutton, S. (1998). Predicting and explaining intentions and behaviour: How well are we doing? Journal of Applied Social Psychology, 28, 1317-1338.

Terry, D.J., y O'Leary, J.E. (1995). The theory of planned behaviour: The effects of perceived behavioural control and self-efficacy. British Journal of Social Psychology, 34, 199-220.

Trafimow, D., Sheeran, P., Conner, M., y Finlay, K.A. (2002). Evidence that perceived control is a multidimensional construct: Perceived control and perceived difficulty. British Journal of Social Psychology, 41, 101-121.

van den Eijnden, R.J.J.M., Spijkerman, R., y Engels, R.C.M.E. (2006). Relative contribution of smoker prototypes in predicting smoking among adolescents: A comparison with factors from the Theory of Planned Behavior. European Addiction Research, 12, 113120.

White, K.M., Terry, D.J., y Hogg, M.A. (1994). Safer sex behavior: The role of attitudes, norms, and control factors. Journal of Applied Social Psychology, 24, 21642192.

Wilkinson, D., y Abraham, C. (2004). Constructing an integrated model of the antecedents of adolescent smoking. British Journal of Health Psychology, 9, 315-333. 